\title{
NIVELES SÉRICOS DE TRIGLICÉRIDOS Y COLESTEROL EN CABALLOS PERUANOS DE PASO BAJO DOS SISTEMAS DE CRIANZA
}

\author{
Triglycerides and Cholesterol Serum Levels in Peruvian Paso Horses \\ Under Two Rearing Systems
}

\author{
César Díaz C. ${ }^{1,2}$, Elmer Plaza C. ${ }^{1}$ y Pedro Chimoy E. ${ }^{3}$
}

\section{Resumen}

El objetivo del presente estudio fue comparar los niveles de triglicéridos (TG), colesterol total (CT) y fraccionado en caballos peruanos de paso (CPP) de la Región Lambayeque. Se analizaron sueros de 50 CPP (25 sedentarios, 25 activos). Los TG y CT se determinaron con el uso de kits comerciales, mientras que las lipoproteínas HDL-C y LDL-C se determinaron después de precipitación selectiva con polímeros precipitantes selectivos (sulfato de dextrán, $\mathrm{Mg}++$ ). Las VLDL-C se calcularon restando al CT los valores de HDL-C + LDL-C. Los niveles promedio fueron de $25.69 \mathrm{mg} / \mathrm{dl}$ para TG, 108.71 $\mathrm{mg} / \mathrm{dl}$ para CT, $66.55 \mathrm{mg} / \mathrm{dl}$ para HDL-C, $21.38 \mathrm{mg} / \mathrm{dl}$ para LDL-C, y $20.78 \mathrm{mg} / \mathrm{dl}$ para VLDLC. Los valores séricos de HDL-C en CPP activos fueron estadísticamente superiores que en los sedentarios $(\mathrm{p}<0.01)$, en tanto que los valores de TG fueron inferiores en caballos castrados en comparación con las yeguas $(\mathrm{p}<0.05)$. Los CPP sedentarios tuvieron niveles menores de HDL-C que los activos, aunque estos últimos tienen menor riesgo aterogénico; sin embargo, tanto caballos activos como sedentarios son poco susceptibles a sufrir hipertrigliceridemia.

Palabras clave: triglicéridos, colesterol total, HDL-C, LDL-C, VLDL-C, Caballos Peruanos de paso

\section{AbStract}

The objective of the study was to compare the level of triglycerides (TG), total cholesterol (TC) and its fractions (VLDC-C, LDL-C and HDL-C) in blood serum of Peruvian Paso Horses (CPP) in the Lambayeque Region. Serum of 50 CPP animals (25 sedentary and 25 active) were analyzed. TG and TC levels were determined using commercial kits, whereas HDL-C and LDL-C were determined using selective polymer precipitants (dextran sulphate, $\mathrm{Mg}++$ ). VLDL-C was calculated by subtracting HDL-C + LDL-C values from TC levels. Mean values were $25.69 \mathrm{mg} / \mathrm{dl}$ for TG, $108.71 \mathrm{mg} / \mathrm{dl}$ for TC, $66.55 \mathrm{mg} / \mathrm{dl}$ for HDL-C, $21.38 \mathrm{mg} / \mathrm{dl}$ for LDL-C, and $20.78 \mathrm{mg} / \mathrm{dl}$ for VLDL-C. HDL-C serum values in active CPP animals were statistically higher than in sedentary horses $(\mathrm{p}<0.01)$, whereas TG values

\footnotetext{
${ }^{1}$ Facultad de Medicina Veterinaria, ${ }^{3}$ Facultad de Ciencias Biológicas, Universidad Nacional Pedro Ruiz Gallo de Lambayeque

${ }^{2}$ E-mail: cf_diaz496@yahoo.com
} 
were statistically lower in castrated horses as compared to mares $(\mathrm{p}<0.05)$. Sedentary horses registered lower levels of HDL-C as compared with active animals, but the latter have lesser atherogenic risk; however, both active and sedentary horses have low susceptibility to get hypertrigliceridemia.

Key words: triglycerides, total cholesterol, HDL-C, LDL-C, VLDL-C, Peruvian Paso Horse

\section{INTRODUCCIÓN}

La crianza del Caballo Peruano de Paso (CPP) ha cambiado en el tiempo, de una crianza tradicional en libertad a una confinación del caballo en el establo, condición que le ocasiona inconvenientes como reducido ejercicio natural y exceso de peso para conformaciones óseas no compatibles para su edad (Mazzi, 2003). En algunos casos, luego del ejercicio, se puede observar cansancio, frecuencia respiratoria elevada, frecuencia cardiaca incrementada, e incluso la muerte, por lo que estos sucesos podrían estar asociados a niveles altos de triglicéridos (TG) y colesterol que conducen a problemas cardiovasculares.

Los niveles de TG en caballos saludables y en ayuno son $<200.17 \mathrm{mg} / \mathrm{dL}$ (Naylor et al., 1980), los cuales son más bajos que el reportado para ponis $(732.47 \mathrm{mg} / \mathrm{dL}$ $1801.51 \mathrm{mg} / \mathrm{dL}$, Bauer, 1983) y asnos (375.54 $\pm 49.6 \mathrm{mg} / \mathrm{dL}$, Forhead et al., 1994). La elevación de TG puede ser de importancia clínica como signo catabólico que demuestra un progreso claro de daño severo del órgano, como es el caso de lipidosis hepática o infiltración grasa de otros órganos. Diferencias notables en hipertrigliceridemia podrían relacionarse con el tipo de equino y sus diferencias en el metabolismo energético y endocrino, así como su respuesta al tipo de dieta y ejercicio físico (Dunkel y McKenzie, 2003). Entre los factores predisponentes para hipertrigliceridemia e hipercolesterolemia están la ingesta reducida de alimento, la influencia del síndrome de respuesta inflamatoria sistémica, así como la azotemia y la gestación avanzada (Dunkel y Mckenzie,
2003), aunque también se observa aumento de los triglicéridos y cortisol en respuesta al ejercicio (García et al., 1999).

Los lípidos son insolubles en agua, por lo que se requiere la formación de complejos lipoproteicos para su transporte en la sangre (Murray et al., 2004). Las lipoproteínas se forman al combinar el colesterol y los triglicéridos con fosfolípidos y apolipoproteínas. Las cuatro principales fracciones lipoproteicas son: HDL-C (lipoproteínas de alta densidad, ?), LDL-C (baja densidad, ?), VLDL-C (muy baja densidad, pre-?) e IDL-C (densidad intermedia, ?-flotadora). El rol del HDL-C es el transporte reverso de colesterol desde los tejidos hacia el emuntorio biliar, por lo que son consideradas como «factor de protección» del riesgo aterogénico, en tanto que las LDL-C están involucradas en el denominado transporte directo del colesterol, que lo distribuye y deposita en los tejidos, incluyendo las paredes vasculares. Además de la vía clásica de transporte inverso de colesterol, se conoce que HDL-C transfiere colesterol a VLDL-C y LDL-C a cambio de triglicéridos (Leaf, 1995).

Dado que ninguna especie es exactamente igual a otra en su metabolismo lipídico, se ha propuesto agruparlas en dos grandes patrones, en base a características similares (Bauer, 1997). El patrón LDL-C (LDL-C > HDL-C) incluye a las especies donde la mayor parte del CT es transportado por LDL-C (seres humanos adultos, cerdos, monos, conejos, cobayos, hámsters y otros de vida silvestre). El patrón HDL-C (HDL-C > LDLC) involucra a los animales donde la mayor parte del colesterol total (CT) es transportado por HDL-C (bovinos, equinos, caninos, 
felinos, ratones y ratas). El ser humano recién nacido respondería al patrón HDL-C, resistente a la aterogénesis. En años recientes, se ha logrado que ratones transgénicos cambien su patrón HDL-C por LDL-C, por lo que desarrollan aterosclerosis (Bauer, 1997; Coppo, 2001). Para llegar a diagnósticos de dislipidemias no es suficiente conocer la tasa de CT, sino esclarecer el tipo de lipoproteína que lo está transportando. En la actualidad es rutina medir la cantidad de colesterol ligado a lipoproteínas de alta densidad (HDL-C) y de baja densidad (LDL-C) (Angel y Angel, 1997).

El presente estudio se llevó a cabo para determinar los valores normales de triglicéridos y colesterol en el CPP, así como establecer si el tipo de crianza y el sexo, influyen significativamente en los valores lipídicos.

\section{Materiales y Métodos}

El presente estudio se llevó a cabo en varios criaderos de caballos de la región Lambayeque, localizada en la zona nor-occidental del Perú. Esta región es de clima cálido, con una temperatura anual promedio de $22.3^{\circ} \mathrm{C}$ (máxima de $33{ }^{\circ} \mathrm{C}$ y mínima de $17{ }^{\circ} \mathrm{C}$ ).

Se trabajó con 50 Caballos Peruanos de Paso clínicamente saludables (13 potros, 15 capones y 22 yeguas), con edades entre 3 y 15 años. La mitad de los animales fueron considerados como caballos activos, ya que realizaban caminatas diarias de $8 \mathrm{~km}$, eran criados en pasturas naturales, donde recibían 4 $\mathrm{kg}$ de una mezcla a base de maíz y polvillo de arroz, además de panca de maíz ad libitum. La otra mitad fueron considerados como caballos sedentarios, ya que realizaban caminatas de $8 \mathrm{~km}$ cada tres días y eran criados en boxes donde recibían $4 \mathrm{~kg}$ de concentrado, 12 $\mathrm{kg}$ de alfalfa verde y $6 \mathrm{~kg}$ de heno de alfalfa.

Se colectaron muestras de sangre (10 ml) por venopunción yugular, empleando tubos al vacío (Vacutainer $\left.{ }^{\circledR}\right)$, a los caballos en reposo entre 07:00 o 08:00, previo ayuno de $12 \mathrm{~h}$. Se dejó sedimentar la sangre durante 20 min, expuesta al sol y a temperatura ambiente, para luego trasladarla en termos refrigera$\operatorname{dos}\left(8^{\circ} \mathrm{C}\right)$ al laboratorio para su análisis.

Los triglicéridos se determinaron por el método enzimático descrito por Fossati y Prencipe (1982) con el kit TG color-Wiener. El CT fue determinado según Allain et al. (1974) con el kit Colestat-Wiener. Las lipoproteínas de alta densidad (HDL-C) fueron separadas precipitando selectivamente las lipoproteínas LDL-C y VLDL-C con sulfato de dextran (PM 50.000) en presencia de iones $\mathrm{Mg}^{++}$. Las lipoproteínas de baja densidad (LDL-C) fueron separadas a través de precipitación selectiva con polímeros de alto peso molecular (sulfato de polivinilo disuelto en polietilenglicol 600). El colesterol ligado a las LDL-C fue determinado restando al CT el valor del sobrenadante. Las lipoproteínas de muy baja densidad (VLDLC) fueron obtenidas restando los valores de HDL-C y LDL-C al CT. Las lecturas para TG y colesterol se realizaron a $505 \mathrm{~nm}$ en espectrofotómetro Teco Diagnostics TC 84.

Para el análisis de los datos se consideró como variables independientes a los tipos de crianza (activos y sedentarios) y como variables dependientes a los niveles de TG, CT, HDL-C, LDL-C, VLDL-C. Se empleó el programa XLStat 2006 para las estadísticas simples, el análisis de varianza y la prueba de Tukey. En todos los casos se consideró un $\mathrm{a}=3 \%$.

\section{Resultados y Discusión}

Los valores de TG, CT y lipoproteínas se presentan en el Cuadro 1. Los valores séricos de HDL-C en caballos activos fueron estadísticamente superiores a los valores de caballos sedentarios ( $\mathrm{p}<0.01$ ), en tanto que los niveles de TG séricos fueron significativamente diferentes entre capones $\mathrm{y}$ yeguas $(\mathrm{p}<0.05)$. 
Cuadro 1. Valores ${ }^{1}$ de triglicéridos (TG), colesterol total (CT) y lipoproteínas $(\mathrm{mg} / \mathrm{dl})$ en Caballos Peruanos de Paso, según el sexo y el tipo de actividad

\begin{tabular}{lccccc}
\hline Parámetro & TG & CT & HDL-C & LDL-C & VLDL-C \\
\hline $\begin{array}{l}\text { Tipo de actividad } \\
\text { Activo (25) }\end{array}$ & $23.15 \pm 9.80$ & $117.55 \pm 33.54$ & $75.44 \pm 21.81$ & $18.64 \pm 11.14$ & $23.45 \pm 11.58$ \\
$\quad$ Sedentario (25) & $28.23 \pm 11.00$ & $99.86 \pm 23.52$ & $57.66 \pm 12.59$ & $24.12 \pm 11.78$ & $18.10 \pm 11.83$ \\
Sexo & & & & & \\
$\quad$ Potro (13) & $28.73 \pm 14.65$ & $114.97 \pm 33.40$ & $72.04 \pm 23.13$ & $23.20 \pm 13.98$ & $19.73 \pm 10.32$ \\
$\quad$ Capón (15) & $19.78 \pm 9.17$ & $103.51 \pm 17.21$ & $64.26 \pm 15.25$ & $18.02 \pm 8.78$ & $21.22 \pm 12.03$ \\
$\quad$ Yegua (22) & $27.55 \pm 7.88$ & $111.25 \pm 34.72$ & $66.28 \pm 21.18$ & $22.62 \pm 12.44$ & $22.34 \pm 12.73$ \\
\hline $\begin{array}{l}\text { Total } \\
\text { Rango máximo y }\end{array}$ & $25.69 \pm 10.62$ & $108.71 \pm 30.03$ & $66.55 \pm 19.78$ & $21.38 \pm 11.68$ & $20.78 \pm 11.89$ \\
\hline mínimo & 50.40 & $43.00-203.2$ & $25.9-129.0$ & $2.08-51.46$ & $1.36-46.59$ \\
\hline
\end{tabular}

${ }^{1}$ Promedio \pm desviación estándar

Los valores séricos obtenidos para TG $(25.69 \pm 10.62 \mathrm{mg} / \mathrm{dl})$ en el CPP son bastante menores a los reportados para caballos de otras razas $(<200.17 \mathrm{mg} / \mathrm{dl}$, Naylor, 1982) y asnos (375.54 mg/dl, Forhead et al., 1994). En las razas Andaluz, Morgan, Árabe, Friesian, Quarter Horse, Thoroughbred, Warmblood y Shire se reporta una media de $844.96 \mathrm{mg} / \mathrm{dl}$ con un rango de 546.48 a $1,619.95 \mathrm{mg} / \mathrm{dl}$ (Coppo et al., 2003). Estas diferencias pueden deberse a la raza y sugieren que los CPP tienen metabolismo aeróbico y riesgo mínimo de desarrollar hipetrigliceridemia.

Si bien se observó diferencia significativa de TG entre capones y yeguas, independientemente del tipo de crianza, los valores encontrados no implican riesgo de lipemia. En la hiperlipemia el promedio de TG es de $2,250 \mathrm{mg} / \mathrm{dl} \pm 1,603 \mathrm{mg} / \mathrm{dL}$ en ponis y caballos miniatura (Mogg y Palmer, 1995) y de $1,470 \pm 168 \mathrm{mg} / \mathrm{dl}$ en asnos (Forhead et al., 1994).
Los promedios de CT en CPP (108.71 $\pm 30.03 \mathrm{mg} / \mathrm{dl}$ ) fueron semejantes a los obtenidos en otras razas equinas $(90-180 \mathrm{mg} / \mathrm{dl}$, Angel y Angel, 1997; 80-140 mg/dl, Coppo, 2001). Los mayores niveles de CT en CPP activos con relación a los sedentarios podrían deberse a la retroinhibición que controla el nivel de CT plasmático y que es inversa a la ingesta de grasa dietaria.

Los valores de HDL-C en CPP (66.55 $\pm 19.78 \mathrm{mg} / \mathrm{dL}$ ) fueron similares a los obtenidos en caballos mestizos de criollo, de pura sangre y cuarto de milla en Argentina (Coppo, 2001) y en Colombia (70 - 140 mg/dl, Angel y Angel, 1997). Los niveles significativamente mayores de HDL-C sérico en CPP activos indicarían que el tipo de crianza estabulado de los CPP sedentarios estaría afectando negativamente su metabolismo lipídico, ya que reciben dietas ricas en energía y proteína, y realizan poco ejercicio físico, por lo que podría cambiar su patrón lipídico, si estos factores se acentúan. 
Los niveles de LDL-C $(21.38 \pm 11.68$ $\mathrm{mg} / \mathrm{dl}$ ) no registraron diferencias significativas respecto al tipo de crianza y sexo, y fueron ligeramente inferiores a los reportados en la literatura $(30.00 \pm 16.00 \mathrm{mg} / \mathrm{dl}$, Coppo et al., 2003). Las concentraciones de VLDL$\mathrm{C}$ en CPP no pudieron ser comparados porque no existen datos en la literatura. Los bajos niveles de LDL-C y VLDL-C sumados no alcanzan el valor de las HDL-C. Así mismo, los bajos niveles de TG permiten afirmar que los CPP tienen un bajo riesgo cardiovascular.

Se puede concluir que los valores de TG en el CPP son bajos en comparación a los valores reportados en caballos de otras razas, por lo que el riesgo de desarrollar hipertrigliceridemia o hiperlipemia es mínimo. El nivel de CT está dentro del rango de otras razas equinas y el riesgo de sufrir aterosclerosis es mínimo por los valores altos de HDL-C, parámetro afectado por el tipo de crianza. Por último. Así mismo, no hubo diferencia en los valores de LDL-C y VLDL$\mathrm{C}$ entre caballos activos y sedentarios con respecto al tipo de crianza y sexo.

\section{Agradecimientos}

A los señores criadores de caballos peruanos de paso Sr. Oscar Vílchez, Sr. José Vásquez, Ing. Hernán Luna Durán, Ing. Manfred Zoeger Navarro; al Criadero de Caballos Peruanos de Paso de la Unión de Cervecerías Peruanas Backus \& Johnston S.A. Así mismo, al Dr. Germán Gorbitz y a la Empresa Agroindustrial Gandulez por proporcionar sus caballos para este estudio, al Blgo. $\mathrm{Mg}$. Jorge Chanamé por su ayuda en el análisis estadístico y al Dr Luis Destefano Beltrán (UPCH) por la revisión del abstract.

\section{Limeratura Citada}

1. Allain CC, Poon LS, Chan CS, Richmond W, Fu PC. 1974. Enzymatic determination of total serum cholesterol. Clin Chem 20: 470-475.

2. Angel G, Angel M. 1997. Interpretación clínica del laboratorio. $5^{\circ}$ ed. Bogotá: Panamericana. $664 \mathrm{p}$.

3. Bauer JE. 1983. Plasma lipids and lipoproteins of fasted ponies. Am J Vet Res 44: 379-384.

4. Bauer JE. 1997. Metabolismo comparado de lípidos y lipoproteínas. Pet's Ciencia 13: 362-376.

5. Coppo JA. 2001. Fisiología comparada del medio interno. Buenos Aires, Argentina: Ed. Dunken, 297 p.

6. Coppo NB, Coppo JA, Lazarte MA. 2003. Intervalos de confianza para colesterol ligado a lipoproteínas de alta y baja densidad en suero de bovinos, equinos, porcinos y caninos. Rev Vet 14: $1-10$.

7. Dunkel B, Mckenzie HC. 2003. Severe hypertriglyceridaemia in clinically ill horses: diagnosis, treatment and outcome. Equine Vet J 35: 590-595.

8. Forhead AJ, French J, Ikin P, Fowler JN, Dobson H. 1994. Relationship between plasma insulin and triglyceride concentrations in hypertriglyceridaemic donkeys. Res Vet Sci 56: 389-392.

9. Fossati P, Prencipe L. 1982. Serum triglycerides determined colorimetrically with an enzyme that produces hydrogen peroxide. Clin Chem 28: 2077-2080.

10. García M, Guzmán R, Cabezas I, Merino $V$. 1999. Evaluación del entrenamiento tradicional del caballo criollo chileno de rodeo mediante el análisis de variables fisiológicas y bioquímicas sanguíneas. Arch Med Vet 31(2): 167-176.

11. Leaf $A D$. 1995. Guía para el tratamiento de dislipidemias. $2^{\text {la }}$ ed. Santafé de Bogotá, Colombia: D'Vinni Ed.

12. Mazzi M. 2003. Caballo de «Andar» Peruano de Paso. En: LVIII Concurso Nacional Oficial del Caballo Peruano de Paso. Lima: Asociación Nacional de Criadores y Propietarios de Caballos Peruanos de Paso.

13. Mogg TD, Palmer JE. 1995. Hyperlipidemia, hyperlipaemia and 
hepatic lipidosis in American miniature horses: 23 cases (1990-1994). J Am Vet Med Ass 207: 604-607.

14. Murray RK, Mayes PA, Granner DK, Rodwell VW. 2004. Harper Bioquímica Ilustrada. $16^{\circ}$ ed. México: El Manual Moderno. $751 \mathrm{p}$.
15. Naylor JM, Kronfeld DS, Acland H. 1980. Hyperlipemia in horses: effects of undernutrition and disease. Am J Vet Res 6: 899-905.

16. Naylor JM. 1982. Hyperlipemia and hyperlipidemia in horses, ponies and donkeys. Comp Cont Educ Pract Vet 4: 321-326. 